\title{
La Côte-Saint-André
}

ZAC du Rival-Olagnières

Stéphane Bleu

\section{(2) OpenEdition}

Journals

Édition électronique

URL : http://journals.openedition.org/adlfi/3448

ISSN : 2114-0502

Éditeur

Ministère de la culture

Référence électronique

Stéphane Bleu, "La Côte-Saint-André », ADLFI. Archéologie de la France - Informations [En ligne], RhôneAlpes, mis en ligne le 01 mars 2009, consulté le 03 mai 2019. URL : http://journals.openedition.org/ adlfi/3448

Ce document a été généré automatiquement le 3 mai 2019.

(c) Ministère de la Culture et de la Communication, CNRS 


\title{
La Côte-Saint-André
}

\author{
ZAC du Rival-olagnières
}

\section{Stéphane Bleu}

Identifiant de l'opération archéologique : 10192

Date de l'opération : 2009 (EX)

\section{Phase 1}

1 Une campagne d'évaluation archéologique a été menée à La ZAC du Rival-Olagnières à la périphérie immédiate du lieu où il fut découvert à la fin du XIX ${ }^{\mathrm{e}} \mathrm{s}$. un char processionnel en bronze, aujourd'hui présenté au Musée de la Civilisation gallo-romaine de Lyon. Cette découverte et une prospection récente réalisée en 2008 (Gonin, 2008) confirment l'extrême sensibilité archéologique du secteur. Seules les parcelles 51p à 55p, de la section cadastrale ZK, ont été sondées. Lors de cette évaluation, quatre-vingt-onze sondages ont été réalisés sur l'ensemble de l'emprise, atteignant une profondeur maximum de $2 \mathrm{~m}$ pour une surface approximative de $2746,14 \mathrm{~m}^{2}$, soit 9,6\% de l'emprise. Les sondages ont atteint un substrat sableux brun-rouille englobant un cailloutis calcaire (terrasse fluvioglaciaire).

2 Il ressort de l'étude stratigraphique une séquence répétitive et relativement simple : la terre végétale recouvre le terrain fluvio-glaciaire altéré d'une trentaine de centimètres d'épaisseur qui présente une matrice limono-argileuse de couleur orangé-rouille, et le terrain fluvio-glaciaire non altéré constitué par une matrice de sables grossiers de couleur grise ou brun-rouille avec présence des graviers, de cailloutis et de galets compris entre $0,05 \mathrm{~m}$ et $0,25 \mathrm{~m}$.

3 Au terme de cette opération, 15 sondages ont permis de mettre en évidence des témoins d'une petite occupation médiévale du $\mathrm{X}^{\mathrm{e}}$ s.-XII ${ }^{\mathrm{e}} \mathrm{s}$. Il s'agit notamment d'une sépulture, de treize fosses de forme circulaire, ovalaire ou parfois irrégulière (rejet domestique ?), d'un 
trou de poteau et d'un empierrement linéaire repérés dans une quinzaine de sondages. Ces structures n'ont livré que quelques artefacts (fragments de tuile GR, nodules de terre cuite et quelques rares tessons de céramique). Seule la fosse a livré quelques pierres chauffées, des fragments de terrre cuite, une terre noire très charbonneuse et plusieurs fragments de céramique attribuables entre les $\mathrm{X}^{\mathrm{e}} \mathrm{s}$. et Xlle $\mathrm{s}$.). Dans un autre sondage, une structure linéaire (empierrement réalisé à l'aide de galets et de nombreux fragments de tegulaeet d'imbrices) d'axe ouest-est, repérée sur une douzaine de mètres de longueur, correspond éventuellement à la fondation d'un mur de clôture (limite de parcelle?) d'époque médiévale ou encore à un drain.

4 Afin de lever toute ambiguïté concernant un éventuel lien entre la sépulture découverte lors de ce diagnostic et les tumuli présents dans le secteur, une chronodate a été réalisée sur un échantillon du squelette. La sépulture est datable entre la seconde moitié du VIle $\mathrm{s}$. et la seconde moitié du X $\mathrm{X}^{\mathrm{e}} \mathrm{s}$. (1210 $\pm 50 \mathrm{BP}$, soit 670-960 après J.-C. en années réelles), sans pic de probabilité (d'après Ch. Oberlin, Laboratoire de ${ }^{14} \mathrm{C}$ ).

\section{Seconde phase}

5 Une seconde campagne d'évaluation archéologique a porté sur le lieu de découverte d'un char processionnel en bronze. Les parcelles 50p, 84p, 85p, 356p de la section cadastrale ZK. Lors de cette évaluation, 161 sondages d'une profondeur maximum de $1,50 \mathrm{~m}$ ont été réalisés. Au total, une surface approximative de $4444.67 \mathrm{~m}^{2}$ aura été ouverte, soit 6,8 \% de l'emprise. Les sondages ont atteint un substrat sableux brun-rouille englobant un cailloutis calcaire (terrasse fluvio-glaciaire).

6 Malgré son apparente monotonie topographique due à une terrasse fluvio-glaciaire würmienne, le site montre une topographie légèrement ondulée favorable à un certain enfouissement des vestiges, qui peut atteindre au grand maximum $0,70 \mathrm{~m}$. Les inondations du Rival, pouvant se produire encore de nos jours (Gonin, 2008), semblent en grande partie responsables des dépôts plus limoneux que l'on peut trouver sur le site, piégés dans des dépressions très peu profondes et recouvrant plus ou moins les vestiges. Ces couches sont malheureusement peu épaisses et parfois peu distinctes. Elles ne permettent l'élaboration d'une stratigraphie relative fiable (mais qui pourrait être tentée lors d'une fouille éventuelle). Un ancien cours du Rival est possible en limite nord du secteur A. Il devrait traverser l'emprise du futur diagnostic de phase 3.

7 Ces sondages ont permis de mettre en évidence les témoins de deux occupations, l'une de l'âge du Bronze et l'autre de la période médiévale ( $\mathrm{X}^{\mathrm{e}} \mathrm{s}$.-XII ${ }^{\mathrm{e}} \mathrm{s}$.).

8 En ce qui concerne la période protohistorique, six structures sub-rectangulaires à pourtour rubéfié disposées en alignement, ont été observées dans le secteur nord-ouest de l'emprise. Elles ne contenaient que peu de mobilier et sont interprétées comme des foyers à pierres chauffantes. Il a toutefois permis de proposer une datation relativement précise grâce à la découverte d'un tesson de céramique non tournée (Bronze final Ilb). Elles ont la même forme (fosses sub-rectangulaires à fond probablement plat et angles arrondis) et leurs dimensions (entre 1,60 m et 1,80 m de longueur et $0,75 \mathrm{~m}$ et 1,60 m de largeur) sont homogènes. Les parois sont vraisemblablement verticales, souvent rubéfiées et parfois durcies par la chaleur. La profondeur conservée n'a pas été perçue lors de ce diagnostic. Leur stratigraphie est toujours la même dans les niveaux inférieurs. Ils sont constitués par la couche de combustible, assez peu épaisse ( $0,05 \mathrm{~m}$ observé en coupe), un 
limon très charbonneux avec quelques éléments partiellement brûlés (brandons). Les niveaux suivants sont composés de galets (quartzite?) pris dans un limon charbonneux. Ces galets portent souvent des traces de chauffe et semblent surtout se trouver au sommet de la couche. La quantité de galets par structures représente une partie importante du volume complet de la structure. La taille des galets reste moyenne $(0,10 \mathrm{~m}$ par 0,20 m).

Depuis ces dix dernières années, ces fours à pierres chauffantes sont bien connus dans le nord de la vallée du Rhône et le Massif Central. Les premières découvertes remontent à la fin des années 80 et s'inscrivent principalement dans le cadre d'opérations préventives. À Montalieu-Vercieu, Isère (Vital, 1991), le gisement de Chalépont a été parmi les premiers sites à livrer ce type de structures, en contexte culturel du premier âge du Fer (VIlle s. av. J.-C.). Dans cet exemple, les structures de combustion sont associées à des structures annexes: petite construction sur poteaux porteurs à Chalépont et vaste complexe d'habitat au Pré de la Cour (structures de combustion ; fosses polylobées ; zones de rejets, etc.).

En ce qui concerne notre opération, il semble éventuellement possible de rattacher les structures découvertes à la nécropole du Premier âge du Fer (char de la Côte-Saint-André) localisée à une centaine de mètres tout au plus (voir à ce sujet: Beeching, Vital, Dal Pra, 1985 : la structure de Soyons, la Brégoule pourrait être mise en relation avec des pratiques cultuelles voire funéraires). Dans le même ordre d'idées, nous pouvons penser que les structures sont le reflet de manifestation(s) d'importance, à la fin de l'âge du Bronze. Dans cette perspective, nous nous situons dans le schéma de grands rassemblements festifs, nécessaires à l'équilibre socio-économique et politique des communautés qui ont vécu ici au cours du VIlle siècle avant notre ère.

Quelques indices d'occupation de la fin de la Protohistoire (La Tène finale) et/ou du début de l'époque gallo-romaine (tessons de céramique grise savonneuse et de sigillée) ont également été identifiés, sans que l'on puisse les rattacher à des structures particulières.

La période médiévale, quant-à-elle, regroupe deux ensembles (zone des bâtiments 1 et 2, zones de constructions légères) dont la morphologie recouvre, semble-t-il, une chronologie différente. Le premier ensemble correspond à deux bâtiments à solins de pierres, orientés nord-sud, dont l'un (le bâtiment 2) livre des traces d'activité métallurgique (forge) à sa périphérie immédiate. Situé à une cinquantaine de mètres du précédent, le deuxième ensemble semble bien différent, livrant des constructions légères (structures excavées qui pourraient éventuellement être des fonds de cabane, des trous de poteau et des fosses).

13 Un vaste établissement rural a donc été mis en évidence au nord de l'emprise (secteurs B et C), représenté par au moins deux bâtiments distincts qui pourraient correspondre à deux unités fonctionnelles. Le bâtiment 1 (complet?) est rectangulaire $\left(52,52 \mathrm{~m}^{2}\right)$ comportant un espace principal $\left(35,24 \mathrm{~m}^{2}\right)$ et un volume secondaire $\left(17,28 \mathrm{~m}^{2}\right)$ qui correspondrait à une galerie ou une annexe. Les murs solins sont constitués de galets assemblés à la terre, parementés et fourrés de galets et de pierres de plus petit module. Leur largeur varie de 0,30 $\mathrm{m}$ à $0,60 \mathrm{~m}$ d'épaisseur. Seules deux assises de galets sont conservées. Le sol est, semble-t-il, en terre battue (US 63 ?) ou peut-être constitué d'un empierrement (?) de cailloutis calcaire observé le long du mur du bâtiment.

14 Le bâtiment 2, quant-à-lui, n'a été dégagé que partiellement. Deux solins de galets identiques à ceux du bâtiment 1 , ont été observés délimitant au sud et à l'ouest une vaste 
salle d'une superficie minimum de $35,84 \mathrm{~m}$. Les traces d'un artisanat du fer ont été identifiées à proximité. Une cinquantaine de scories représentant une dizaine de $\mathrm{kg}$ de déchets ont été ramassées dans une fosse à une dizaine de mètres au sud-est du bâtiment. Quelques scories ont également été ramassées dans une fosse située au centre de la pièce. Les scories sont relativement légères, fragiles et petites. Quatre fonds de calotte (ou culots) ont aussi été identifiés (diamètre de $0,10 \mathrm{~m}$ environ et poids compris entre $200 \mathrm{~g}$ et $300 \mathrm{~g}$ ). Les foyers correspondants à cet artisanat n'ont pas été formellement identifiés. Ces éléments indiquent qu'une activité de forge a été menée là ou à proximité. Il semble que le premier ensemble ait été utilisé durant le haut Moyen Âge ( $\mathrm{V}^{\mathrm{e}} \mathrm{s}$.-VIII ${ }^{\mathrm{e}} \mathrm{s}$.).

A proximité immédiate du bâtiment 1 à envrion une trentaine de mètres à l'est, un autre ensemble est constitué de structures beaucoup plus légères. Il s'agit de cinq grandes fosses correspondant peut-être à des structures excavées de type fonds de cabane (aucun négatif de trous de poteau), de fosses charbonneuses, de fosses et/ou trous de poteau (certains très proches de ceux observés au Châtelard à Chirens). Le mobilier associé à ce deuxième ensemble est attribuable à l'An Mil, entre le $\mathrm{X}^{\mathrm{e}} \mathrm{s}$. et le Xlle $\mathrm{s}$. Un tesson est proche des éléments de la fin du haut Moyen Âge pendant la période carolingienne.

Enfin, cinq tranchées à galets ont également été identifiées. La plupart sont localisées au nord de l'emprise. Ces structures se retrouvent dans le secteur concerné par la présence des bâtiments. Il est actuellement encore bien difficile de déterminer la nature de ces tranchées: vestiges de drains ou de fossés comblés, de limites parcellaires (manses? emplacements de haies?) ou de clôture (murs d'enclos ?). Leur axe semble le même que celui des bâtiments. Ce sont, en tous cas, les seules traces conservées de délimitation de l'espace.

Rappelons enfin la découverte d'une sépulture isolée, en limite sud de l'emprise et à une cinquantaine de mètres du bâtiment 1 , lors de la phase 1 . Un squelette de femme âgée y reposait allongé sur le dos dans une simple fosse creusée dans la terrasse.

\section{INDEX}

Thèmes : céramique, forge, fosse, foyer, galet aménagé, habitat, sépulture, trou de poteau, tuile operation expertise (EX) Index chronologique : Âge du Bronze, IXe siècle apr. J.-C., Moyen Âge, VIIe siècle apr. J.-C., VIIIe siècle apr. J.-C., Xe siècle apr. J.-C., XIe siècle apr. J.-C., XIIe siècle apr. J.-C.

Index géographique : Rhône-Alpes, Isère

\section{AUTEUR}

STÉPHANE BLEU 